\title{
Scanning SQUID-on-tip Magnetic and Thermal Microscopy
}

\author{
Kousik Bagani \\ Department of Condensed Matter Physics, Weizmann Institute of Science, Rehovot 7610001, Israel \\ E-mail address: kskbgn@gmail.com \\ https://doi.org/10.54162/SD01-25201/01
}

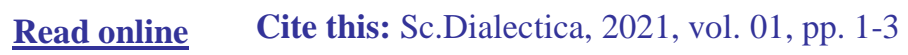

Keywords: Superconducting Quantum Interference Device (SQUID)

\begin{abstract}
Scanning magnetic and thermal imaging using Superconducting Quantum Interference Device (SQUID) fabricated on the apex of a sharp tip has attracted great attention because of its record magnetic sensitivity, thermal sensitivity and nanoscale spatial resolution. Many interesting phenomena like vortex dynamics in a superconductor, quantum hall state, and heat dissipation in graphene etc. has been investigated using scanning SQUID on tip microscopy. This is one of the most powerful tool for the investigation of a wide variety of quantum systems and novel materials.
\end{abstract}

\section{Introduction}

Superconducting quantum interference device (SQUID) is a superconducting (SC) loop having two Josephson junctions (Fig. 1a). The electrical properties of such a device can be modulated by applied magnetic flux $(\Phi)$ through the loop with a universal period called the "flux quantum", $\left(\Phi_{0}\right)$ which enables the use of a SQUID as magnetometers (Fig. 1b). In fact, SQUID is the most sensitive device available for measuring the magnetic field and has been extensively used in research like measuring magnetic susceptibility, small current, voltage, studying biological samples, superconducting qubits etc. Due to its extreme sensitivity to the magnetic field, lots of efforts have been given in the development of scanning SQUID microscopy for magnetic imaging to investigate the microscopic magnetic structure and interaction and to explore many interesting phenomena [1]. However, there are technical limitations to reduce the size of the SQUID sensor and to scan very close proximity above the surface of a sample; scanning SQUID microscopy is lacking special resolution and sensitivity. Recently, Prof. Eli Zeldov's group at the Weizmann Institute of Science has developed a novel technique to fabricate the world's smallest SQUID on the apex of a sharp tip, which iscapable of nano-scale magnetic imaging [2-5]. The SQUID-on-tip (SOT) sensors can be fabricated as small asa few tens of nanometers and the geometry of the SOT allows scanning very close proximity to the surface resulting in high sensitive magnetic imaging with nano- scale spatial resolution. As the magnetic field strength of a dipole decreases rapidly with distance, the ability to scan very close proximity to the surface results in SOTs into extremely sensitive scanning devices with less than single electron spin sensitivity. Recently, a novel nano-scale thermal imaging technique has been developed using the SQUID-on-tip [6-8]. Existing thermal imaging techniques are mostly not compatible with the low-temperature operation. In contrast, a nano-thermometer based on SOT is suitable for cryogenic thermal imaging and it is the world's most sensitive thermometer at cryogenic temperature with submicro-Kelvin temperature sensitivity. Scanning SOT is one of the most powerful tools for the investigation of a wide range of electronic systems, quantum states of matter and novel devices due to its nanoscale spatial resolution, record magnetic, thermal sensitivity and operation at elevated magnetic fields.

\section{SQUID-on-tip Fabrication}

The fabrication SQUID-on-tip (SOT) starts with pulling a hollow quartz tube with an outer diameter of $1 \mathrm{~mm}$ to form a pair of sharp pipettes with a tip diameter controllable between 30 to $400 \mathrm{~nm}$ [2]. Then a superconductor is deposited on the sharp hollow pipette from three different angles (Fig. 1c) which results in a superconducting loop at the apex which is connected to two superconducting leads (Fig. 1d and 1e). Where the leads make contact with the ring, superconducting regions are "Strong", while in contrast, the two parts of the ring in the gap between the leads constitute two weak links forming the SQUID at the apex of the tip (Fig. 1g). Here, the two Josephson junctions of the SQUID are two weak links in the form of Dayem bridges. The SQUID-on-tip is then attached with a quartz tuning fork which is used as a topography sensor guiding the tip to hover above very close proximity 
a

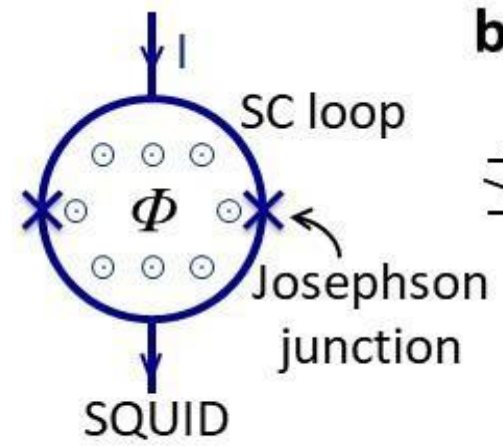

C

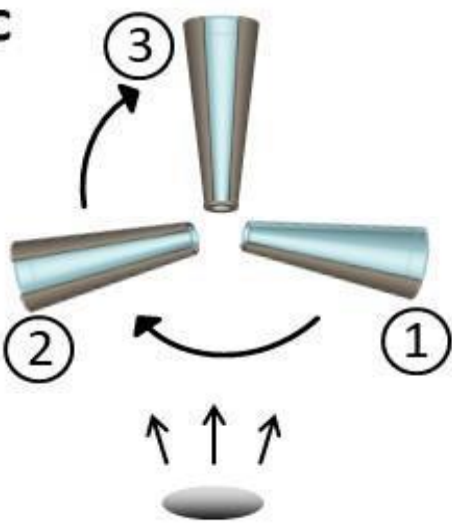

Thermal deposition

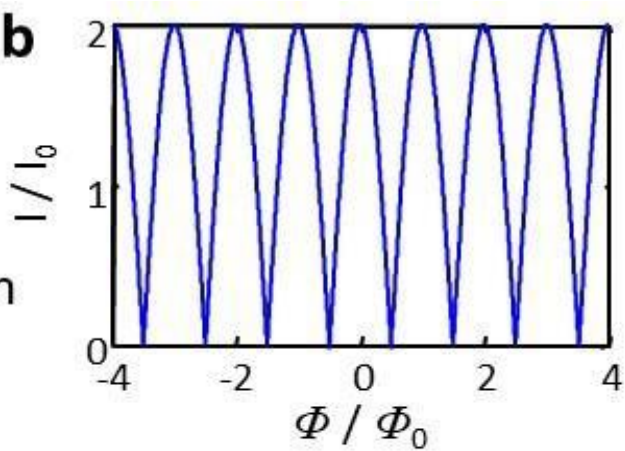

$\mathbf{f}$

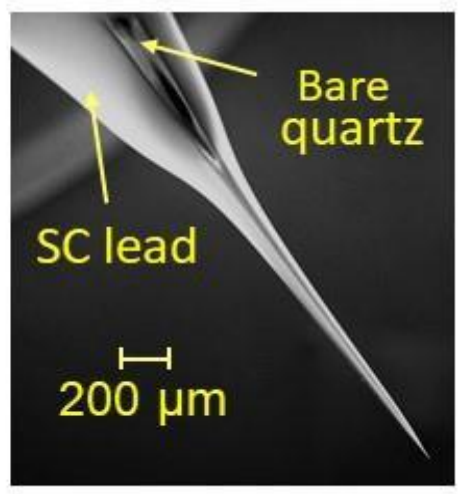

d

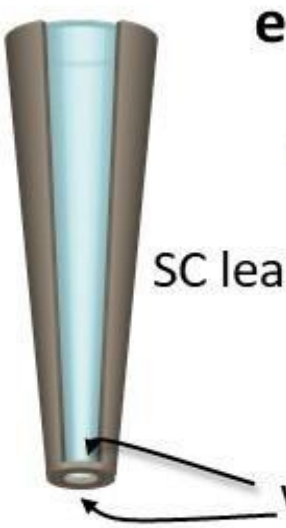

e

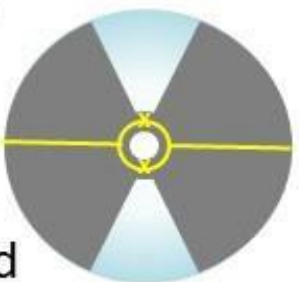

Bottom View

weak links
Side View

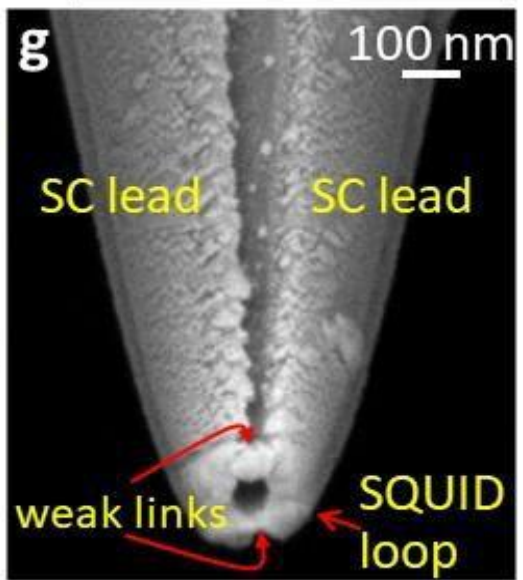

Figure 1. (a) Schematic representation of a SQUID. (b) Oscillation of critical current of a SQUID with applied magnetic flux with a period of one flux quantum. (c) Position of pipettes during three steps deposition of SOT fabrication. (d) and (e)Side and bottom view of schematic drawings of a SQUID-on-tip showing weak link/ Josephson junctions and SQUID formation. (f) Image of a pulled quartz pipette after three steps deposition of a superconductor [2]. (g) SEM image of a SQUID-on-tip showing superconducting loop at the apex with two weak links forming SQUID.

to the surface. SOTs are usually fabricated by thermal deposition with elemental superconductors like Aluminum $(\mathrm{Al})$, Indium (In), Lead ( $\mathrm{Pb})$, Niobium $(\mathrm{Nb})$, Tin $(\mathrm{Sn})$ etc [28]. $\mathrm{Pb} \mathrm{SOT}$ attains record spin sensitivity down to 0.38 $\mu_{\mathrm{B}} / \mathrm{Hz}^{1 / 2}$ [3] and thermal sensitivity better than $500 \mathrm{nK} /$ $\mathrm{Hz}^{1 / 2}$ [6-8]. However, recently a new method has been developed for SOT fabrication using collimated magnetron sputtering that allows versatile use of a wide range of high $\mathrm{H}_{\mathrm{c} 2}$ superconducting materials like Mo-Re, NbN, MoGe, $\mathrm{WSi}, \mathrm{MgB}_{2}$, and possibly even high- $\mathrm{T}_{\mathrm{c}}$ superconductors [9]. MoRe SOTs fabricated using this technique, operates up to an unprecedentedly high magnetic field of $5 \mathrm{~T}$ [9]. This advancement greatly diversifies the applicable superconducting materials for SOT fabrication and thus significantly expands the working regime of nanoscale scanning magnetic and thermal microscopy. Operating in wide ranges of magnetic fields renders the SOT as a powerful scanning imaging technique for investigation of magnetic phenomena and dissipation mechanisms in a wide variety of quantum systems, and novel materials.

Magnetic and Thermal microscopy
Since the development of state-of-art SOT microscopy, it has led to key insights into microscopic properties and vortex dynamics of superconductors, magnetism at oxide interfaces and magnetic topological insulators, etc. [3$5,10]$. Using SOT microscopy, the equilibrium current of individual quantum Hall edge states in graphene has been imaged for the first time and it reveals that the edge states carry a pair of counter propagating currents which was generally understood to carry only a downstream current [11]. The concept of counter propagating currents was predicted in 1994 but since upstream current do not couple to electrical transport, they were completely ignored and forgotten. An induced magnetic monopole response to an electric charge above a quantum Hall electron system has been studied using this newly developed microscopy technique [11]. Furthermore, from the microscopic magnetic image of the twisted bi-layer graphene system, it is revealed that there is spatial variation in twist angle and topographic image of Landau levels in quantum hall state is obtained [12]. Moreover, the SOT microscopy has the potential for exploiting emergent microscopic magnetic properties, current distributions and fundamental aspects in fractional quantum hall state, quantum anomalous hall 
state, van der Waals superconductors, TIs and 2D magnetic materials etc. Very recently, scanning SOT has been demonstrated as the world most sensitive temperature sensor, establishing a new tool of cryogenic thermal imaging [6]. SOT allows studying dissipation processes in quantum systems with nanoscale spatial resolution. Phonon emission from single atomic defects and edges in graphene has been images and studied extensively [7]. Energy dissipation mechanisms in normal and quantum hall edge states have been studied using scanning SOT nano-thermometry [8]. This method should be a novel tool for studying fundamental processes that involve dissipation mechanisms like scattering, electron phonon coupling, decoherence, breaking of topological protection in a wide range of quantum systems and electronic devices.

\section{Acknowledgement}

The author would like to thank Prof. Eli Zeldov for his support and encouragement.

\section{References}

[1] J. R. Kirtley, "Fundamental studies of superconductors using scanning magnetic imaging", Rep. Prog. Phys., 2010, vol. 73 , pp. 126501.

[2] A. Finkler et. al., "Self-Aligned Nanoscale SQUID on a Tip”, 2010, Nano Letters vol. 10, pp. 1046-1049.

[3] D. Vasyukov et. al., "A scanning superconducting quantum interference device with single electron spin sensitivity", Nature Nanotechnol., 2013, vol. 8, pp. 639-644.

[4] A. Uri et. al., "Electrically Tunable Multiterminal SQUID-on-Tip", Nano Letters, 2016, vol. 16, pp. 69106915.

[5] E. O. Lachman et. al., "Visualization of superparamagnetic dynamics in magnetic topological insulators", Science Advance 2015, vol. 1, pp. e1500740.

[6] D. Halbertal et. al., "Nanoscale thermal imaging of dissipation in quantum systems", Nature 2016, vol. 539, pp. 407-410.

[7] D. Halbertal et. al., "Imaging resonant dissipation from individual atomic defects in graphene", Science 2017, vol. 358, pp. 1303-1306.

[8] A. Marguerite, et. al., "Imaging work and dissipation in the quantum Hall state in graphene", Nature 2019, vol. 575, pp. 628-633.

[9] K. Bagani et. al., "Sputtered Mo66Re34 SQUID-on-Tip for High-Field Magnetic and Thermal Nanoimaging”, Phys. Rev. Applied 2019, vol. 12, pp. 044062.
[10] L. Embon et. al., "Imaging of super-fast dynamics and flow instabilities of superconducting vortices", Nature Commun. 2017, vol. 8, pp. 85.

[11] A. Uri et. al., "Nanoscale imaging of equilibrium quantum Hall edge currents and of the magnetic monopole response in graphene", Nature Physics 2020, vol. 16, pp. $164-170$

[12] A. Uri et. al., "Mapping the twist-angle disorder and Landau levels in magic-angle graphene", Nature 2020, vol. 581, pp. 47-52.

\section{Author's biography}

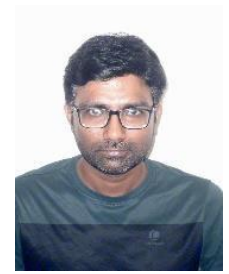

Dr. Kousik Bagani was born at Howrah district of West Bengal, India in the year 1988. $\mathrm{He}$ earned his MSc in Physics and from Jadavpur University, Kolkata in 2010. He did his $\mathrm{PhD}$ in Experimental Condensed Matter Physics from Saha Institute of Nuclear Physics in 2016. He worked as a postdoctoral researcher at Weizmann Institute of Science, Israel from 2016 to 2020. Currently, he is working as postdoctoral research associate at University of Basel, Switzerland. During PhD tenure, his work was focused on studying role of defects on magnetic and thermal properties of graphene and oxide nano materials. During postdoctoral research, he worked on the development of novel scanning SQUID-on-tip microscopy technique. He has published many international journal papers and presented his research work in various national and international conferences. 